\title{
THE BORDISM CLASS OF A QUASI-SYMPLECTIC MANIFOLD
}

\author{
PETER S. LANDWEBER
}

It is not known which unoriented bordism classes contain $S p$ manifolds (the stable tangent bundle admitting a reduction to the symplectic group). As an approximation, a class of smooth manifolds is introduced, containing all $S p$-manifolds and quaternionic projective spaces $H P(n)$, and its image in the unoriented bordism ring $\mathscr{N}$ determined.

Let $\tau(M)$ denote the tangent bundle of a smooth manifold. If $\xi$ is a right quaternionic vector bundle, the conjugate $\xi^{*}$ is a left quaternionic vector bundle; thus for right quaternionic vector bundles $\xi$ and $\eta$, the tensor product $\xi \otimes_{H} \eta^{*}$ is a real vector bundle.

A closed manifold $M$ will be said to be quasi-symplectic if for some $k$ and finite collection of right quaternionic vector bundles $\xi_{i}, \eta_{i}$

$$
\tau(M) \oplus k R=\sum \xi_{i} \otimes_{H} \eta_{i}^{*}
$$

Since $\xi \otimes_{H} H^{*}=\xi_{R}$ is the underlying real bundle of $\xi$, an $S p$-manifold is quasi-symplectic. It is clear that $M \times M^{\prime}$ is quasi-symplectic if $M$ and $M^{\prime}$ are.

ThEOREM. An unoriented bordism class $[M]_{2} \in \mathscr{N}$ contains a quasisymplectic manifold if and only if $[M]_{2}$ is a fourth power in $\Re$.

According to Milnor [3], a class $[M]_{2} \in \mathscr{N}$ is a fourth power if and only if all Stiefel-Whitney numbers of $M$ involving some $w_{i}$ with $i \neq 0(\bmod 4)$ vanish. Moreover, if $[M]_{2}=\left([N]_{2}\right)^{4}$ then $w_{4 i_{1}} \cdots$ $w_{4 i_{r}}[M]=w_{i_{1}} \cdots w_{i_{r}}[N]$. Thus in one direction the theorem follows from

LeMma 1. If $\xi$ and $\eta$ are right quaternionic vector bundles, then $w_{i}\left(\xi \otimes_{H} \eta^{*}\right)=0$ for $i \neq 0(\bmod 4)$.

Proof. By the splitting principle, $\xi$ and $\eta$ may be taken to be quaternionic line bundles. In this case, it follows by the methods of [2] or [4] that

$$
w\left(\xi \otimes_{H} \eta^{*}\right)=1+w_{4}\left(\xi_{R}\right)+w_{4}\left(\eta_{R}\right) .
$$

Moreover, $w\left(\xi \otimes_{H} \xi^{*}\right)=1$ for $\xi$ a quaternionic line bundle; and for any

Received by the editors December 20. 1966. 
right quaternionic vector bundle $\eta, w\left(\eta_{R}\right)$ is the mod 2 reduction of the total (symplectic) Pontrjagin class $p(\eta)$.

Before returning to the proof of the theorem, we prove

Lemma 2. If $M$ is quasi-symplectic and $\xi \rightarrow M$ is a right quaternionic vector bundle, then the associated quaternionic projective space bundle $P(\xi)$ is quasi-symplectic.

Proof. Let $\pi: P(\xi) \rightarrow M$ denote the projection, and $\eta \rightarrow P(\xi)$ the canonical symplectic line bundle over $P(\xi)$. It follows from $[4$, Theorem 1.3] and [2] that

$$
\tau(P(\xi)) \oplus \eta \otimes_{H} \eta^{*}=\pi^{*} \tau(M) \oplus \pi^{*} \xi \otimes_{H} \eta^{*},
$$

hence $P(\xi)$ is quasi-symplectic if $M$ is. In particular, $H P(n)$ is quasisymplectic (take $M$ a point) and

$$
\tau(H P(n)) \oplus \eta \otimes_{H} \eta^{*}=(n+1) \eta_{R}
$$

as is well known.

We now complete the proof of the theorem. Let $\eta \rightarrow R P(n)$ denote the Hopf line bundle and put $R P(m, n)=P(\eta \oplus m R)$; thus $R P(m, n)$ is fibred over $R P(n)$ with fibre $R P(m)$. According to P. Anderson [1], the unoriented bordism ring $\mathscr{N}$ is generated by the classes $[R P(m, n)]_{2}$. In a similar fashion, let $H P(m, n)=P(\zeta \oplus m H)$ with $\zeta \rightarrow H P(n)$ the canonical symplectic line bundle. By Lemma 2, $H P(m, n)$ is quasi-symplectic. In view of (1), (2) and (3), it follows readily as in [1] that

$$
w_{4 i_{1}} \cdots w_{4 i_{r}}[H P(m, n)]=w_{i_{1}} \cdots w_{i_{1}}[R P(m, n)],
$$

hence $[H P(m, n)]_{2}=\left([R P(m, n)]_{2}\right)^{4}$. Since $\mathscr{N}$ is generated by the $[R P(m, n)]_{2}$, every class $\left([M]_{2}\right)^{4}$ of $\mathscr{N}$ contains a quasi-symplectic manifold. Q.E.D.

Remark. Consider quasi-symplectic manifolds $M^{k}$ such that the normal bundle to $M^{k}$ has the form $\eta \otimes_{H} \eta^{*} \oplus \zeta_{R}$ for a quaternionic line bundle $\eta$ and quaternionic bundle $\zeta$. There result bordism groups $' \Omega_{\boldsymbol{k}}^{S p}$, and ${ }^{\prime} \Omega^{S p}=\sum_{\boldsymbol{k}}^{\prime} \Omega_{\boldsymbol{k}}^{S p}$ is a graded left module over the symplectic bordism ring $\Omega^{s p}$. It can be shown that ${ }^{\prime} \Omega^{S p}$ is a free module over $\Omega^{s p}$ on the classes $[H P(n)] \in^{\prime} \Omega_{4 n}^{S p}, n \geqq 0$. Thus the image of ' $\Omega^{S p}$ in $\Re$ consists of the sums of classes $\alpha[H P(2 n)]_{2}=\alpha\left([R P(2 n)]_{2}\right)^{4}$ for $n \geqq 0$ and $\alpha \in \Re$ containing an $S p$-manifold. However the image of $\Omega^{S p}$ in $\mathscr{N}$ is not known.

AdDed in Proof. R. E. Stong has shown that every symplectic manifold of positive dimension less than 24 bounds in the unoriented 
sense. See Some remarks on symplectic cobordism, Ann. of Math. 86 (1967), 425-433.

\section{REFERENCES}

1. P. G. Anderson, Cobordism classes of squares of orientable manifolds, Ann. of Math. (2) 83 (1966), 47-53.

2. W. C. Hsiang and R. H. Szczarba, On the tangent bundle of a Grassman manifold, Amer. J. Math. 86 (1964), 698-704.

3. J. Milnor, On the Stiefel-Whitney numbers of complex manifolds and of spin manifolds, Topology 3 (1965), 223-230.

4. R. H. Szczarba, On tangent bundles of fiber spaces and quotient spaces, Amer. J. Math. 86 (1964), 685-697.

UNIVERSITY OF VIRGINIA 